\title{
EVOLUÇÃO DA COR DURANTE A MATURAÇÃO DAS UVAS TINTAS*
}

\author{
COLOR EVOLUTION DURING MATURATION OF RED GRAPES
}

\author{
Gilberto Massashi Ide**, Carlos Eugênio Daudt***
}

RESUMO

Foram feitos estudos de tonalidade e intensidade de cor na película e na polpa durante o desenvolvimento e a maturação da uva das cultivares Cabernet Sauvignon, Cabernet Franc, Merlot, Pinot Noir Vitis vinifera) e Isabel (Vitis labrusca) nas safras de 1990 e 1991. A tonalidade na película diminuiu acentuadamente no inicio do desenvolvimento das bagas estabilizando-se após a "veraison". As boas condiçoes climáticas (baixa precipitação pluviométrica e umidade, alta insolação) da safra de 1991 tiveram pouca influência sobre a tonalidade. A intensidade de cor na película aumentou durante a maturação da uva e as condiçōes climáticas de 1991 propiciaram maior intensidade nas cultivares Cabernet Sauvignon, Cabernet Franc e Merlot pouco influindo, no entanto, nas cultivares Pinot Noir e Isabel. Na polpa houve estabilidade da intensidade de cor, porém, a tonalidade apresentou-se variável com tendência a estabilizar-se durante a maturaçāo.

Palavras-chave: cor, maturação, uvas tintas.

\section{SUMMARY}

Color hue and color intensity analysis were done on the skin and on pulp of Vitis vinifera Cabernet Sauvignon, Cabernet Franc, Merlot, Pinot Noir, and Vitis labrusca Isabel. Samples were collected weekly from some weeks before veraison to harvest during the years of 1989-1990 and 1991-1992. The skin hue decreased in the beginning, stabilizing after veraison. The good climatic conditions - low rain and humidity, lots of sunshine days - of the 1991 harvest had no influence on the hue. The color intensity of the skin, on the other hand, increased during all the maturation period. In the good climatic conditions of january and february of
1991, Cabernet Sauvignon, Cabernet Franc e Merlot produced more color intensity in the skin what did not happen with Pinot Noir and Isabel. Color intensity in the pulp was stable; the hue, not very stable at the beginning, stabilized later on in the last period of maturation.

Key words: color, maturation, red grape.

\section{INTRODUÇÃO}

A cor é um dos requisitos mais importante na avaliação da qualidade do vinho e, como é sabido, ela provém dos polifenóis, principalmente as antocianinas.

Em nosso país onde a implantação da pesquisa em vitivinicultura e relativamente recente, sente-se a falta de conhecimento da composição das uvas e dos vinhos. A maioria dos conhecimentos são extraídos de pesquisas desenvolvidas na França, Estados Unidos, Austrália, Itália, etc.

As antocianinas, pigmentos vermelhos da uva e do vinho tinto identificados por RIBÉREAULGAYON (1959), apresentam-se na forma monoglicosídicas presentes em Vitis vinifera e diglicosídicas encontradas unicamente em uvas americanas e híbridas (AMERINE et al, 1972).

As antocianinas diglicosídicas são mais estáveis à descoloração do que as correspondentes monoglicosídicas, porém, foi observado que estas são menos propensas a oxidação (ROBINSON et al, 1966).

Sudraud apud GLORIES (1984a) define intensidade de cor (IC) como a soma das densidades óticas dos componentes vermelhos e amarelos medidos a um comprimento de onda de 520 e $420 \mathrm{~nm}$, respectivamente. A tonalidade (T) é a relação das densidades óticas a 420 e $520 \mathrm{~nm}$.

A medida da absorbância do vinho a 420,520 e $620 \mathrm{~nm}$ conduz a um melhor conhecimento da compo-

\footnotetext{
* Parte da Dissertação de Mestrado do primeiro autor conclúdo em março de 1992 no Curso de Pós-Graduação em Ciência e Tecnologia de Alimentos da Universidade Federal de Santa Maria (UFSM).

** Engenheiro de Alimentos, Mestre, Professor do Centro de Ciências Agroveterinárias da Universidade do Estado de Santa Catarina (UDESC). 88520-000 - Lages, SC, Brasil.

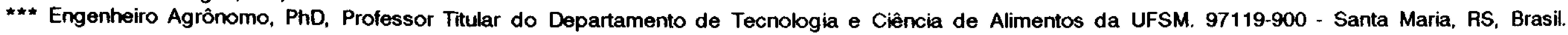


sição da cor do vinho e da sua tonalidade traduzindo de modo satisfatório a sensação visual do degustador (GLORIES, 1984b).

A extração dos polifenóis da película das uvas é descrita por inúmeros pesquisadores. SOMERS (1968), RIBÉREAU-GAYON (1971), DUMAZERT et al (1978), PIRIE \& MULLINS (1980) realizaram a maceração da película, sem trituração, utilizando soluções, tempos, números de extrações e temperaturas de maceração variáveis.

GUILLOUX (1981), DARNE (1988) e GONZALEZSANJOSE et al (1990) realizaram maceraçāo similar ao descrito acima, mas as películas foram previamente liofilizadas e moídas.

ROGGERO et al (1986) e LEE \& JAWORSKI (1987) trituraram as películas das uvas mediante a sua separação e subseqüente extração com solução de metanol.

Qualquer que seja o método, è quase impossível extrair totalmente os polifenóis de um tecido (GUILLOUX, 1981).

O objetivo deste trabalho foi acompanhar a evolução da tonalidade e da intensidade de cor dos grãos de algumas cultivares de uvas tintas do Rio Grande do Sul.

\section{MATERIAL E MÉTODOS}

Foram estudadas uvas das cultivares tintas Cabernet Sauvignon, Cabernet Franc, Merlot, Pinot Noir e Isabel.

Todas as cultivares selecionadas para o estudo eram conduzidas em espaldeiras exceto a Isabel que era conduzida no sistema de latada.

As amostras foram coletadas semanalmente nas safras de 1990 e 1991, pela parte da manhã, a partir do dia 21 de dezembro de 1989 e 20 de dezembro de 1990, respectivamente. Foram coletadas cerca de 200 bagas por cultivar, induzindo a uma obtenção representativa das amostras, entre 15 a 20 plantas selecionadas previamente por cultivar.

As uvas de cada cultivar foram congeladas imediatamente após a coleta e assim mantidas por um período médio de uma semana. Após este período de conservação, ainda sob efeito do congelamento, procedeu-se a retirada da película, manualmente, para extração dos polifenóis mediante trituração usando $75 \mathrm{ml}$ de metanol aquecido a ebulição por 5 minutos em banho maria; após filtração e evaporação do metanol o volume foi completado a $100 \mathrm{ml}$ com solução a $12 \%$. Da polpa previamente triturada e filtrada, os polifenóis foram extraídos utilizando-se $25 \mathrm{ml}$ de polpa adicionados de $50 \mathrm{ml}$ de etanol; após agitação, o líquido ficou em repouso por 30 minutos. Após a filtração, o etanol foi evaporado e o volume completado a $25 \mathrm{ml}$ com água destilada.
A tonalidade e a intensidade de cor foram determinadas medindo-se a absorbância do extrato, acima obtido, a 420 e $520 \mathrm{~nm}$ (GLORIES, 1978) em cubetas de $10 \mathrm{~mm}$ para a polpa e $1 \mathrm{~mm}$ para a película.

A tonalidade foi calculada mediante a relação entre a absorbância medida a $420 \mathrm{~nm}$ e a $520 \mathrm{~nm}$ [1.420/1.520] e a intensidade de cor pela soma das duas absorbâncias [l.420 + 1.520$]$.

\section{RESULTADOS E DISCUSSÃO}

A tonalidade na película da uva, em todas as cultivares, diminuiu acentuadamente até atingir a "veraison" (Figuras 1 e 2 ) observando-se a seguir, uma certa estabilidade. STONESTREET (1965) relacionou a tonalidade como a relação tanino/antocianina e, portanto, a diminuição inicial pode ser interpretada, segundo esse raciocínio, como o aumento significativo de antocianina em relação ao tanino; e o aumento proporcional do teor de tanino em relação a antocianina na película após a "veraison" iustifica a estabilidade da tonalidade.

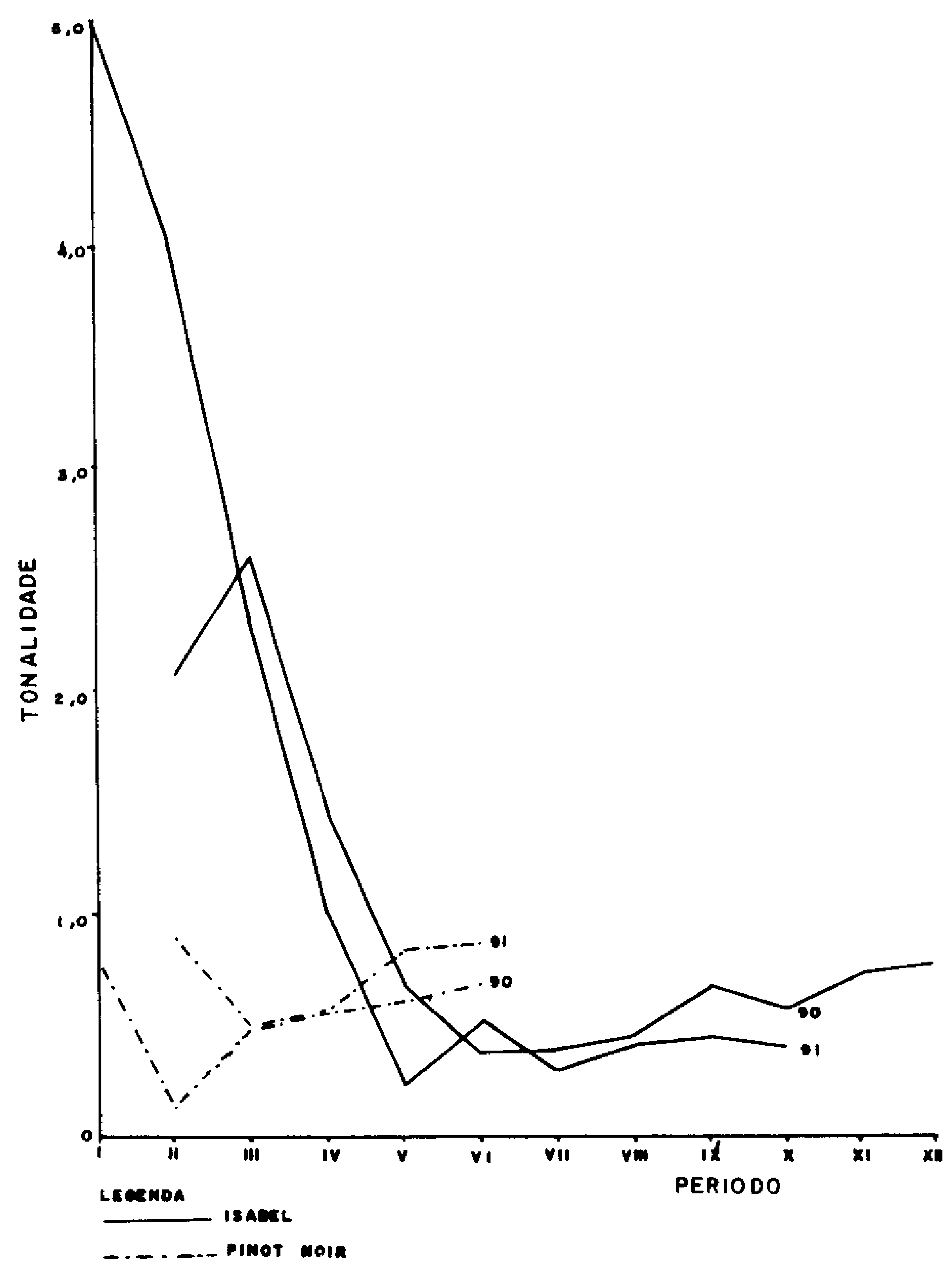

FIGURA 1 - Tonalidade (1.420/1.520) na película das cultivares Isabel e Pinot Noir nas safras de 1990 e 1991. 


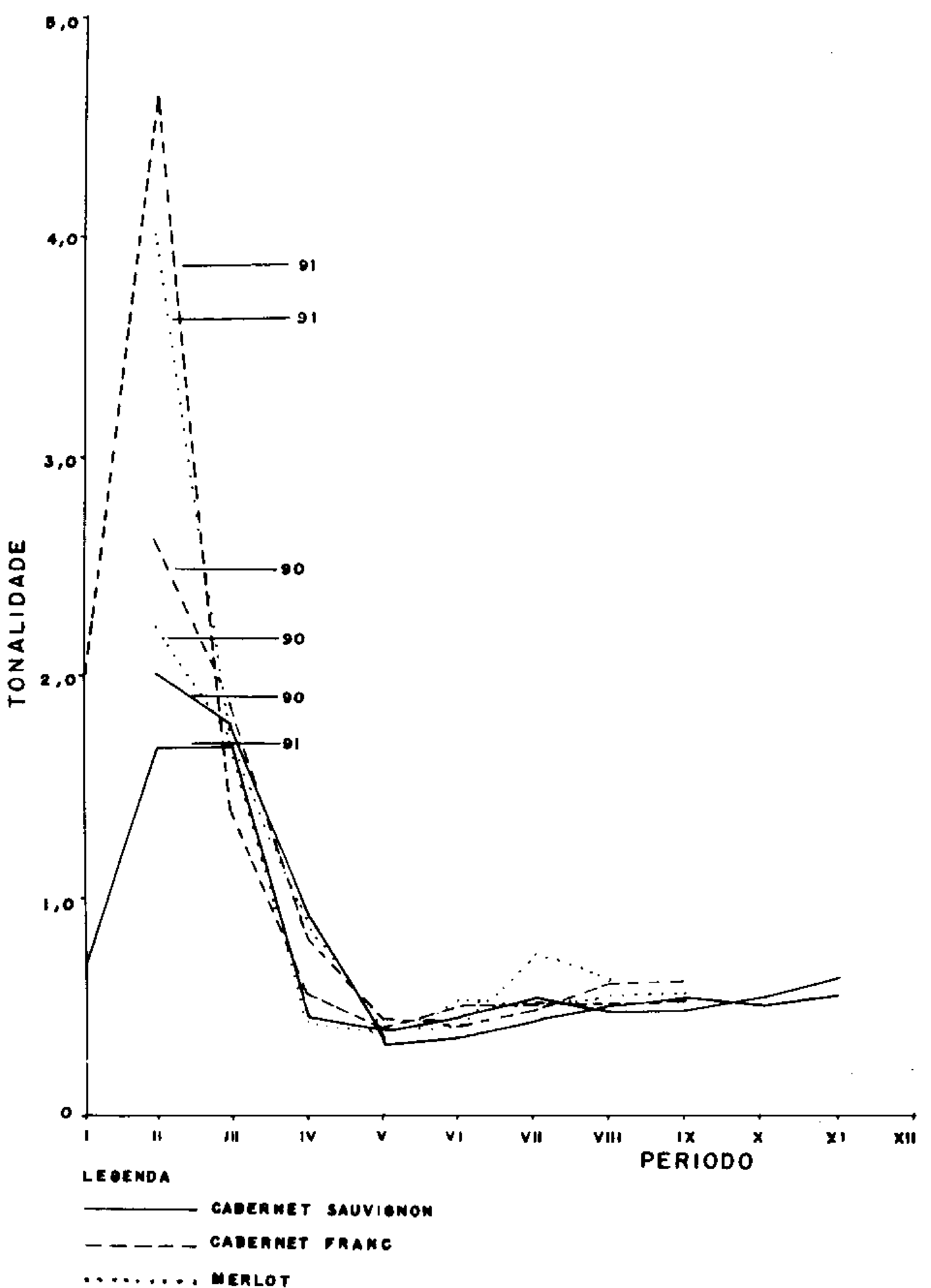

FIGURA 2 - Tonalidade (1. 420/1.520) na película das cultivares Cabernet Sauvignon, Cabernet Franc e Merlot nas safras de 1990 e 1991.

As condições climáticas (baixa umidade, pouca chuva e muita luminosidade) da safra de 1991 não tiveram influência na tonalidade (Figura 2); exceto, porém em pequenas proporções, nas cultivares isabel e Pinot Noir (Figura 1).

A intensidade de cor na película, em todas as cultivares, no decorrer da maturação aumentou, observando-se maior intensidade na safra de 1991 (Figura 3); a exceção foram as cultivares isabel e Pinot Noir (Figura 4). A Isabel apresentou intensidade de cor, em ambas as safras, similares as demais cultivares da safra de 1991; no entanto, se forem considerados em função do peso das bagas (peso da baga de Isabel é $60 \%$ a $80 \%$ maior do que as demais cultivares) esta equivalência torna-se drasticamente reduzida.

O aumento na intensidade de cor, no decorrer da maturação, ocorreu devido ao aumento dos teores de antocianinas e de taninos (IDE, 1992), proporcionando uma idéia do desenvolvimento e da quantidade relativa dos compostos fenólicos da uva, principalmente as antocianinas.

Hoje, os vinicultores brasileiros raramente utilizam tratamento diferenciado no processo produtivo, en-

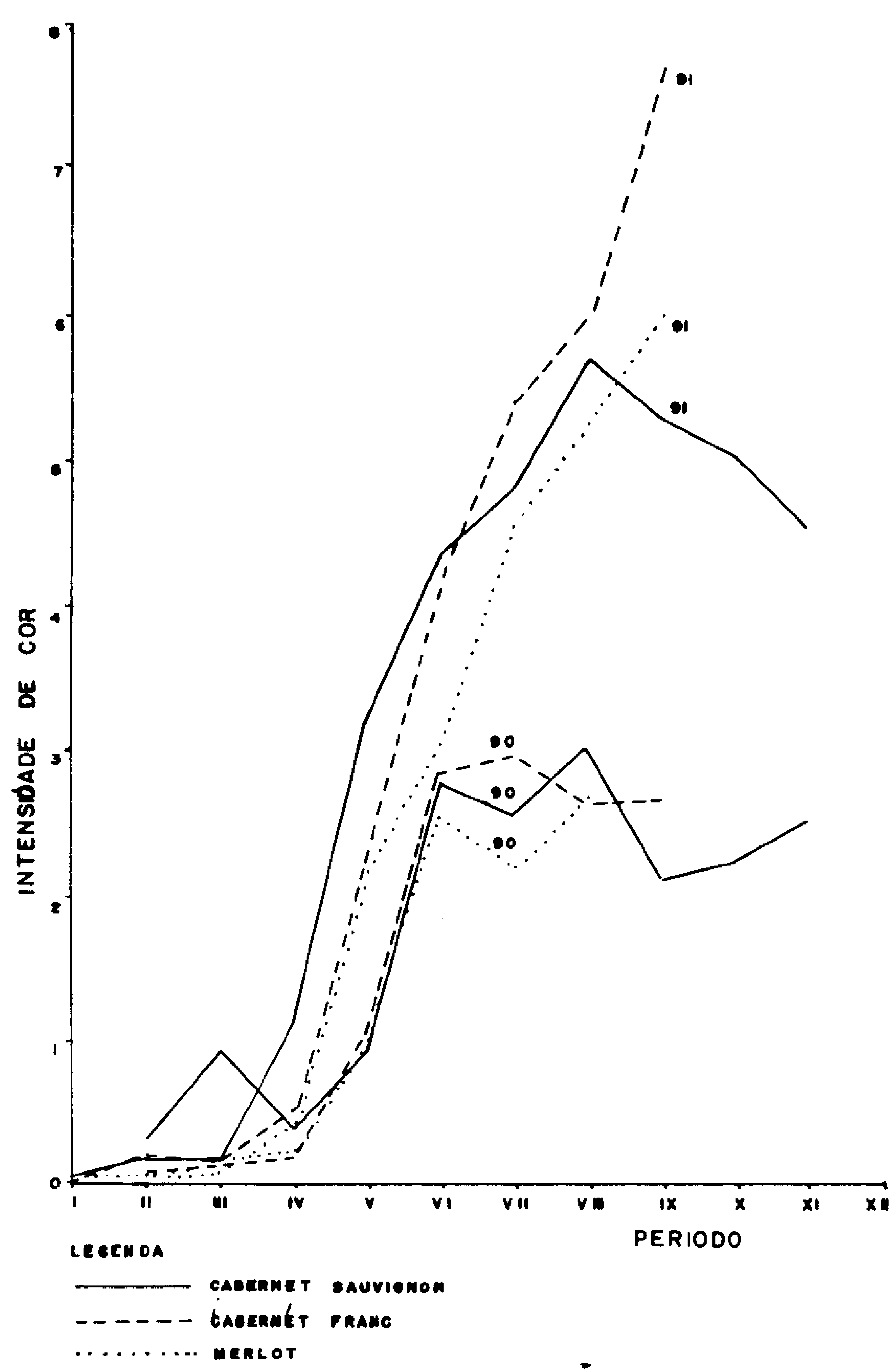

FIGURA 3 - Intensidade de cor $(1.420+1.520)$ na película das cultivares Cabernet Sauvignon, Cabernet Franc e Merlot nas safras de 1990 e 1991.

tre uvas colhidas em safras propícias com as outras, condições essas oscilatórias que ocorrem anualmente e freqüentemente em nosso país.

As uvas, apesar da safra propícia (baixa pluviosidade, umidade e alta insolação) apresentam diferenças nos teores de acidez e sólidos solúveis (brix) pouco significativas em relação à intensidade de cor proveniente do teor de antocianinas; portanto, a intensidade de cor, devido a sua relativa facilidade de determinação, pode vir a colaborar no tratamento adequado que deve ser dado durante a vinificação em uvas, de acordo com as cultivares e 0 ano de safra.

As distorções observadas na cultivar isabel devido ao seu sistema de plantio (latada) e na Pinot Noir, que no Brasil é uma cultivar sabidamente problemática, podem ser vistas e confirmadas pelo desenvolvimento da tonalidade $e$ intensidade de cor durante a maturação (Figura 1 e Figura 4).

$\mathrm{Na}$ polpa, a tonalidade e a intensidade de cor (Figura 5 e Figura 6) manteve-se praticamente constante 


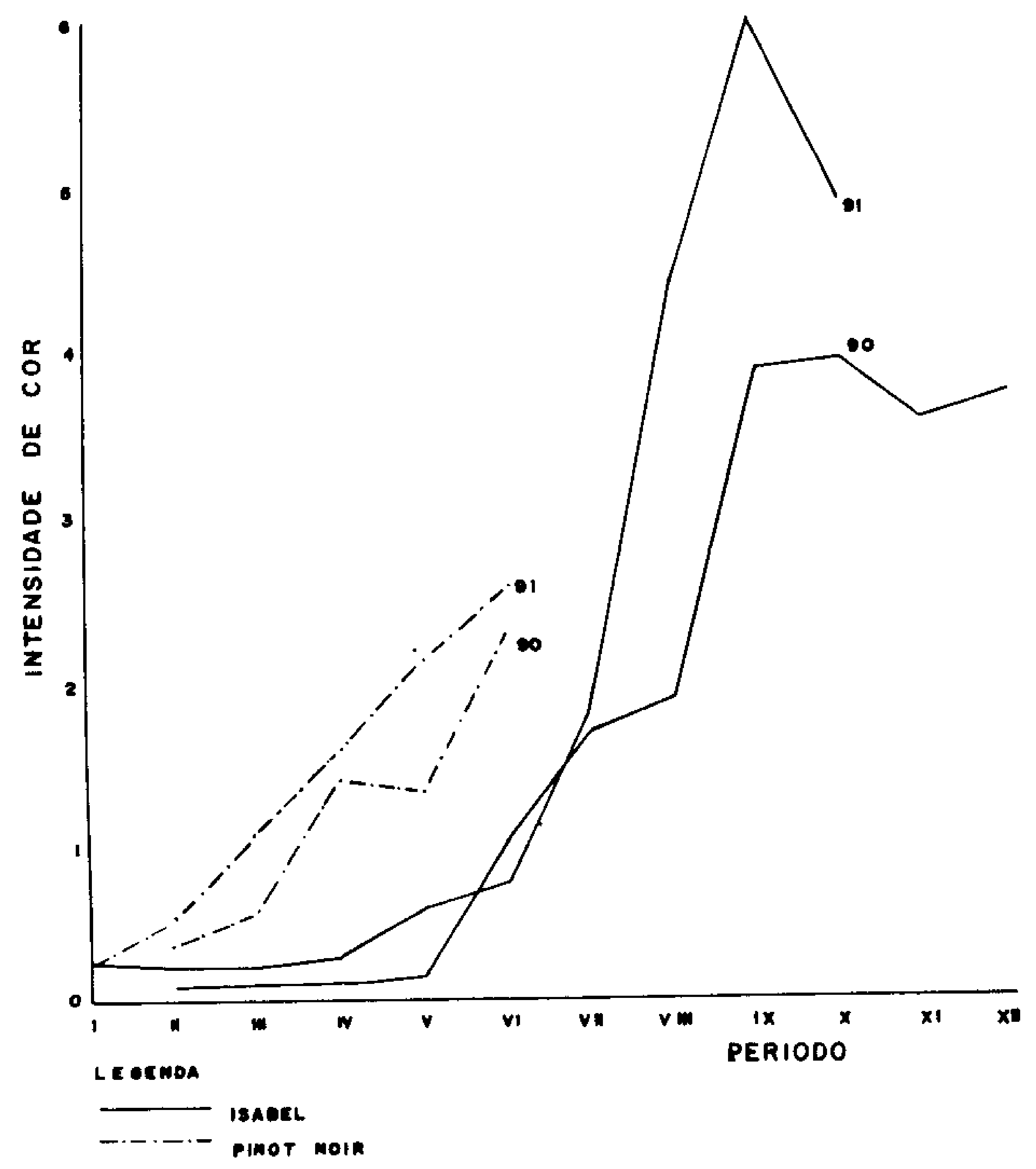

FIGURA 4 - Intensidade de cor $(1.420$ + 1.520) na película das cultivares Isabel e Pinot Noir nas safras de 1990 e 1991.

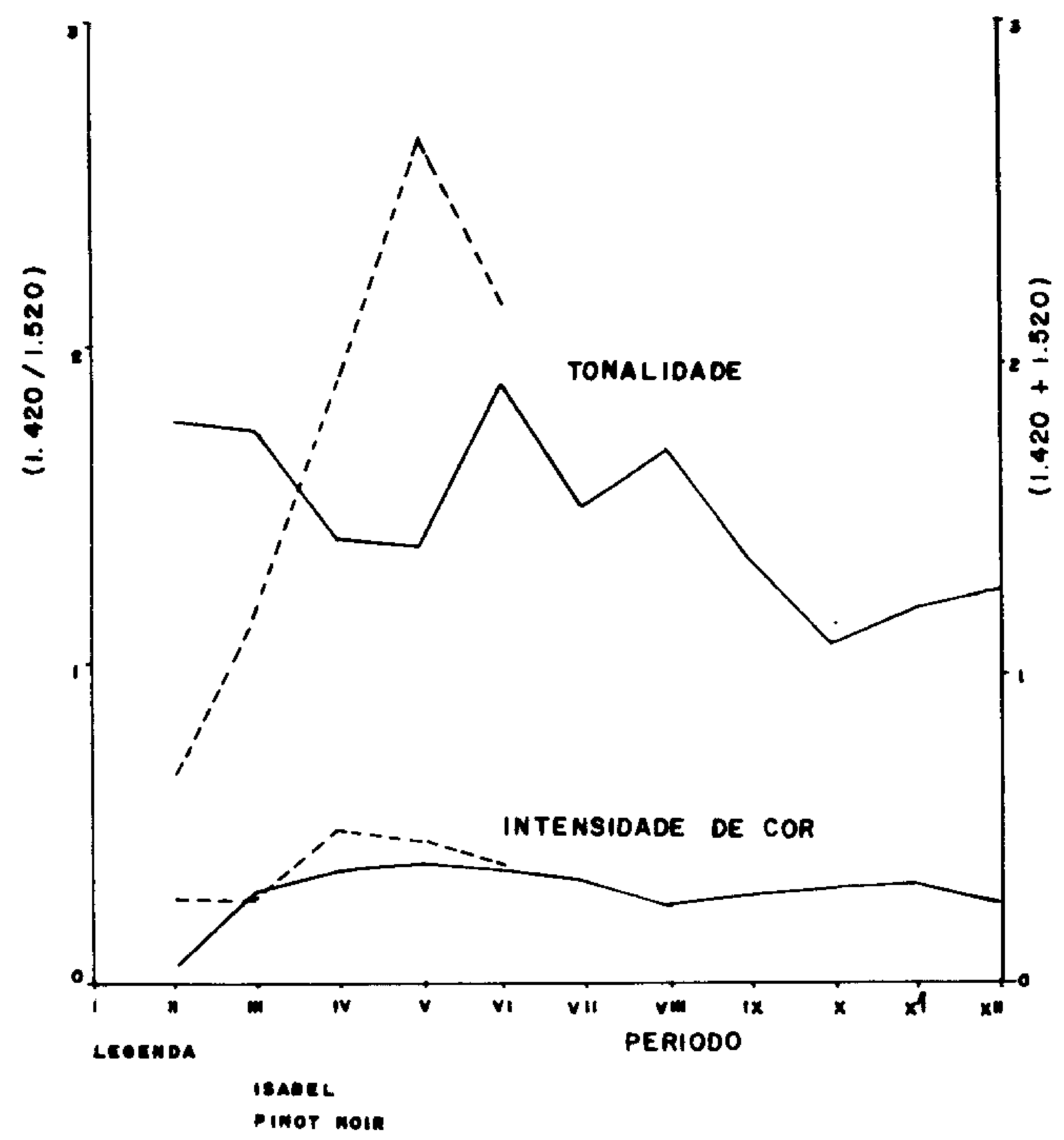

FIGURA 5 - Tonalidade e intensidade de cor na polpa das cultivares Isabel e Pinot Noir na safra de 1990.

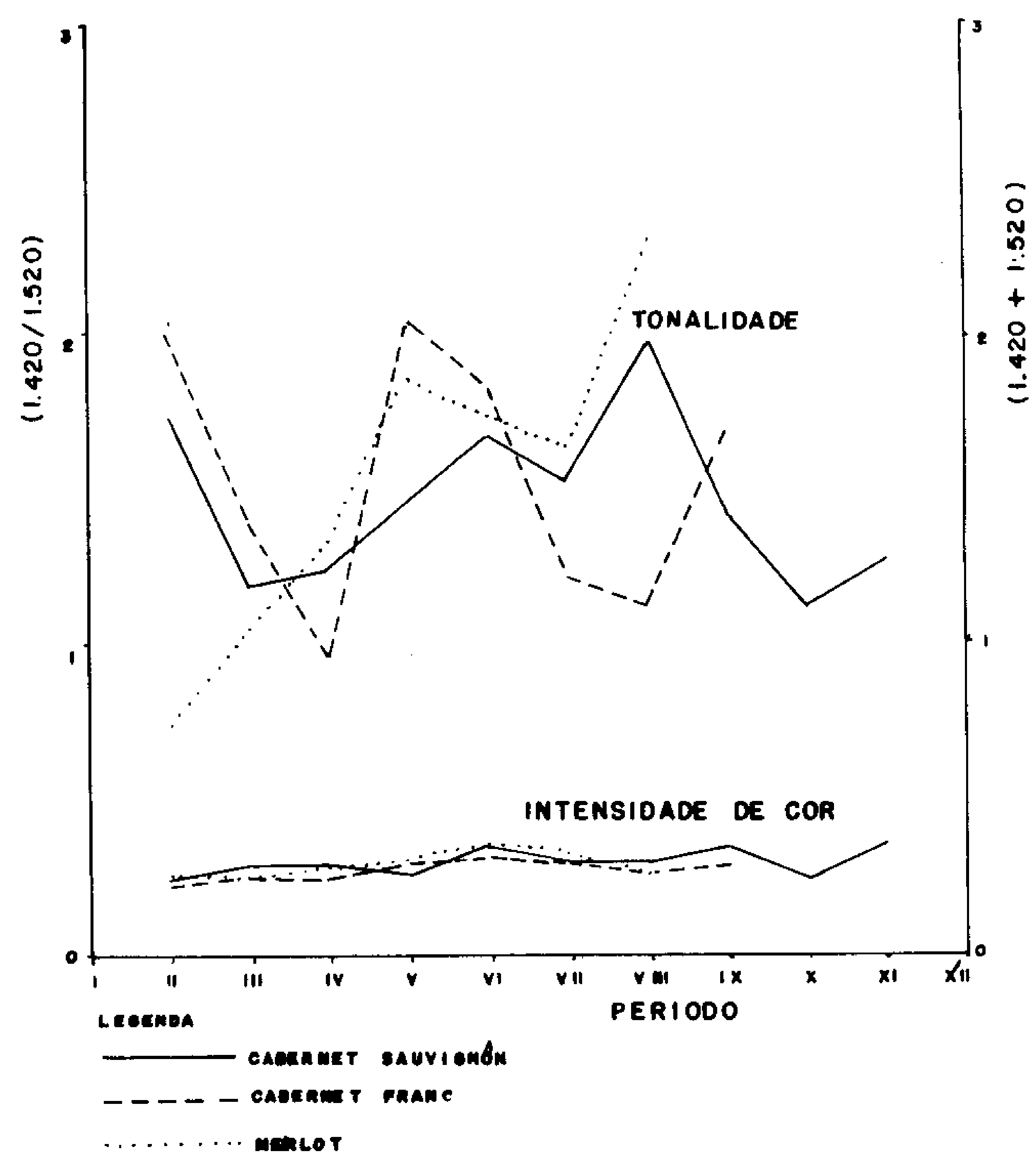

FIGURA 6 - Tonalidade e intensidade de cor na polpa das cultivares Cabernet Sauvignon, Cabernet Franc e Merlot na safra de 1990.

em todas as fases da maturação; as oscilações na tonalidade foram provocadas, provavelmente, pelas antocianinas que foram passadas a polpa no momento da separação da película.

\section{AGRADECIMENTOS}

Os autores agradecem o CNPq pelo parcial financiamento do projeto.

\section{REFERÊNCIAS BIBLIOGRÁFICAS}

AMERINE, M.A., BERG, H.W., CRUESS, W.S. The technology of wine making. 3.ed. New York: AVI Publishing, 1972. $802 \mathrm{p}$.

DARNE, G. Evolution des differentes anthocyanines des pellicules de Cabernet Sauvignon au cours du developpment des baies. Conn Vigne Vin, França, v. 22, n. 3, p. 225-231. 1988.

DUMAZERT, G., MARGULIS, H., MONTREAU, F.R. Evolution des composés phenoliques au cour de la maturation dún Vitis vinifera blanc: Le Mauzac. Ann Technol Agric, v. 22, n. 2, p. 137-151, 1973. 
GLORIES, Y. Evolution des composés phénoliques au cours du vieillisment du vin. Ann Nutr Alim, v. 32, p. 1163-1169, 1978.

GLORIES, Y. La coleur des vins rouges. $1^{\text {a }}$ parte: Les equilibres des anthocyanes et des tanins. Conn Vigne Vin, França, v. 18, n. 3, p. 195-217, 1984a.

GLORIES, Y. La coleur des vins rouges. $2^{a}$ parte: Mesure, origine et interpretation. Conn Vigne Vin, França, v. 18, n. 4, p. 253-271, 1984b.

GONZALEZ-SANJOSE, M.L., BARON, L.J.R., DIEZ, C. Evolution of anthocyanins during maturation of Tempranillo grape variety (Vitis vinifera) using polynomial regression models. J Sci Food Agria, USA. v. 51, p. 337-343, 1990.

GUILLOUX, M. Evolution des composés phénoliques de la grappe pendant la maturation du raisin. Influence des facteurs naturels. Bordeaux, 1981, $125 \mathrm{p}$. Thése (Docteur ès Oenologie) - Université de Bordeaux II, Bordeaux, França, 1981.

IDE, G.M. Evoluçăo dos compostos fenólicos na maturaçăo da uva $\theta$ no tempo de maceração do vinho. Santa Maria, RS, 1992, 87 p. (Dissertação de Mestrado em Ciência e Tecnologia de Alimentos Universidade Federal de Santa Maria).

LEE, C.Y., JAWORSKI, A.W. Phenolic compounds in white grapes grown in New York. Am J Enol Vitic, Davis-USA, v. 38, n. 4, p. 277-281, 1987.
PIRIE, A.J.G., MULLINS, M.G. Concentration of phenolics in the skin of grape berries during fruit development and ripening. Am J Enol Vitic, Davis-USA, v. 31, n. 1, p. 34-36, 1980.

RIBEREAU-GAYON, P. Recherches sur le anthocyanes des vegetaux. Application au genre Vitis. Paris, 1959. Thése (Docteur Sciences Physiques) Libr. genérale de l'enseignement, Paris, 1959.

RIBEREAU-GAYON, P. Evolution des composés phenoliques au cours de la maturation du raisin. 1Experimentation 1969. Conn Vigne Vin, França, v. 5, n. 2, p. 247-261, 1971.

ROBINSON, W.B., WEIRS, L.D., BERTINO, J.J., et al. The relation of anthocyanin composition to color stability of New York state wines. Am J Enol Vitic, USA, v. 17, n. 2, p. 178-184, 1966.

ROGGERO, J.P., COEN, S., RAGONNET, B. High performance liquid chromatography survey on changes in pigment content in ripening grapes of Syrah. An approach to anthocyanin metabolism. Am J Enol Vitic, Davis-USA, v. 37, n. 1, p. 77-83, 1986.

SOMERS, T.C. Pigment profiles of grape and wines. Vitis, v. 7, p. 303-320, 1968.

STONESTREET, E. Contribuition à l'étude des tanins et de la metiere colorante des vins rouges. Bordeaux, 1965. $77 \mathrm{p}$. Thése (Docteur es Oenologie) - Université de Bordeaux II, Bordeaux, França, 1965. 